\title{
The role of stellar feedback in the formation of galactic disks and bulges in a $\Lambda$ CDM Universe
}

\section{Daniel Ceverino and Anatoly Klypin}

\author{
Astronomy Department, New Mexico State University, Las Cruces, NM, USA
}

\begin{abstract}
Although supernova explosions and stellar winds happens at scales bellow $100 \mathrm{pc}$, they affect the interstellar medium (ISM) and galaxy formation. We use cosmological Nbody + Hydrodynamics simulations of galaxy formation, as well as simulations of the ISM to study the effect of stellar feedback on galactic scales. Stellar feedback maintains gas with temperatures above a million degrees. This gas fills bubbles, super-bubbles and chimneys. Our model of feedback, in which $10 \%-30 \%$ of the feedback energy is coming from runaway stars, reproduces this hot gas only if the resolution is better than $50 \mathrm{pc}$. This is 10 times better than the typical resolution in cosmological simulations of galaxy formation. Only with this resolution, the effect of stellar feedback in galaxy formation is resolved without any assumption about sub-resolution physics. Stellar feedback can regulate the formation of bulges and can shape the inner parts of the rotation curve.
\end{abstract}

Keywords. hydrodynamics, methods: numerical, galaxies: formation

Stellar feedback is the most difficult ingredient in galaxy formation models. The basic idea is that massive stars inject energy, mass and metals back to the ISM through stellar winds and supernova explosions. It is crucial to understand where and how the energy from massive stars is released back to the ISM. While a large fraction of massive stars are found in stellar clusters, $10-30 \%$ are found in the field with high peculiar velocities (Gies 1987). The current explanation of these runaway stars is that they were ejected from stellar clusters (Blaauw 1961; Poveda et al. 1967). Besides, the significant fraction of runaway stars, little attention has been paid to their effect on galaxy formation.

We model runaway stars by adding a random velocity to a fraction of the stellar particles, so $\% 10$ of runaway stars have peculiar velocities higher than $40 \mathrm{Km} \mathrm{s}^{-1}$. The heating rate from massive stars is modeled as the rate of energy injection from a single stellar population. This energy release is nearly constant over $40 \mathrm{Myr}$ (Leitherer et al. 1999). The cooling rates must include cooling bellow $10^{4}$ degrees. The conditions of molecular clouds should be included in order to reproduce the effect of stellar feedback in the ISM. We first study the effect of stellar feedback in the ISM, using simulations of a $4 \mathrm{Kpc}$ piece of the ISM with a $14 \mathrm{pc}$ resolution (Figure 1). Then, we check whether this picture holds when the resolution is degraded to the resolution that our cosmological simulations can achieve at high redshift. We find that a 50-pc resolution plus the effect of runaway stars can reproduce a 3-phase ISM (Figure 2). Finally, we study the effect of stellar feedback in galaxy formation at high redshift using cosmological hydrodynamical simulations (Figure 3).

The simulations were performed using the Eulerian gasdynamics + N-body Adaptive Refinement Tree (ART) code (Kravtsov 2003). This code includes metallicity-dependent radiative cooling, UV heating, star formation and stellar feedback. 

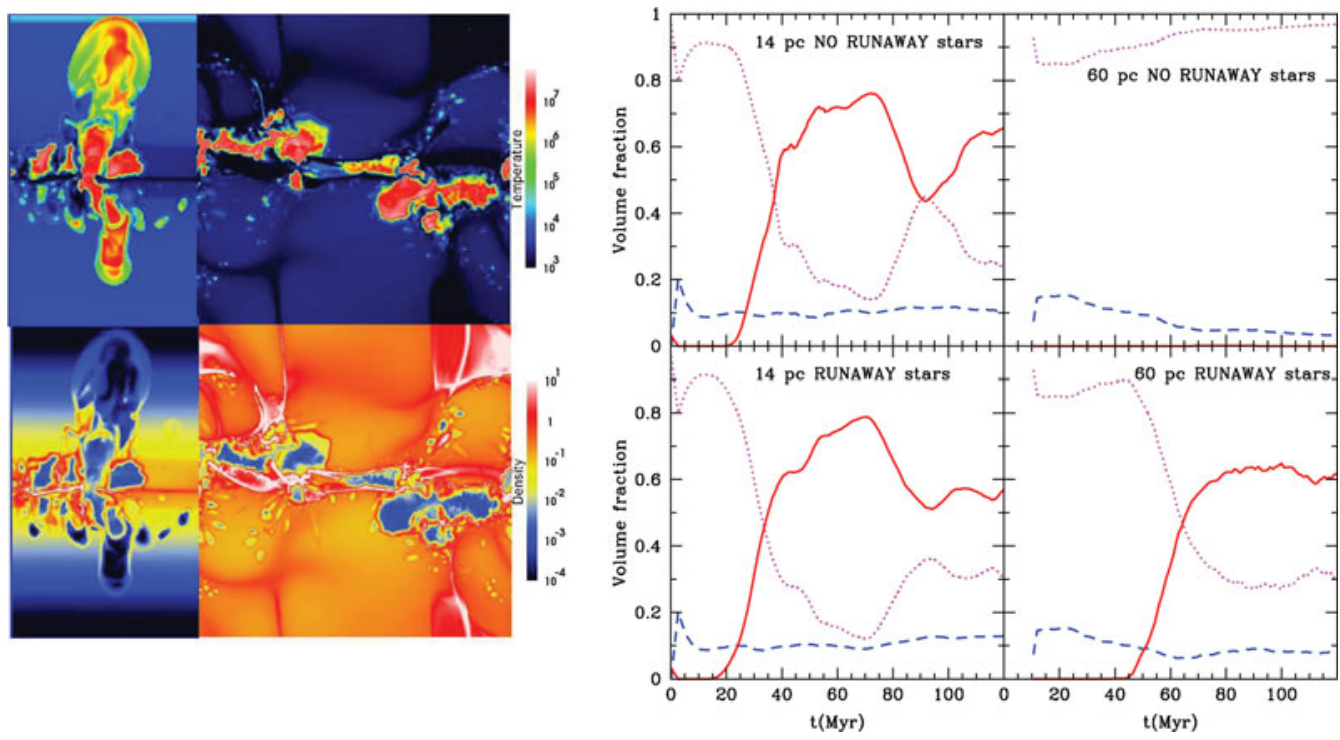

Figure 1. Left panels: Density and temperature in slices perpendicular to a galactic plane (left) and parallel (right). A chimney filled with very hot and diffuse gas is created from the energy input of stellar super-clusters. Small bubbles in the field are the result of the stellar feedback from runaway stars. Right panels: Evolution of the volume occupied by different gas phases: $T<10^{3} K$ (dashed line), $10^{3} K<T<10^{4} K$ (dotted line), and $T>10^{4} K$ (full line). The simulations have a $1 \mathrm{Kpc}$ box with four different models. The hot phase does not develop in the low resolution run without runaway stars (top right panel). If runaway stars are included, the hot phase is recovered even at low resolution. As a result, a fraction of runaway stars changes the thermodynamical conditions of the ISM at low resolutions.
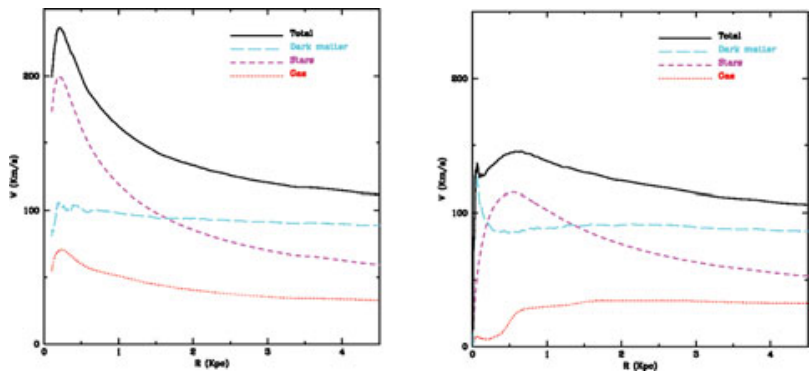

Figure 2. Circular velocity profile of a forming galaxy at redshift 6 for two different resolutions: $120 \mathrm{pc}$ (left) and $60 \mathrm{pc}$ (right). The low-resolution case has the problem of a too massive bulge, which is much weaker in the high-resolution case, where the stellar feedback is more efficient and can regulate the growth of the bulge.

\section{References}

Blaauw, A. 1961, Bull. Astron. Inst. Neth. 15, 265

Gies, D. R. 1987, ApJS, 64, 545

Kravtsov A., 2003, ApJ (Letters) 590, 1

Leitherer, C., et al. 1999, ApJS, 123, 3

Poveda, A., Ruiz, J., \& Allen, C. 1967, Bol. Obs. Tonantzintla Tacubaya, 4, 86 